\title{
Simulation and Model Validation of a Pneumatic Conveying Drying for Wood Dust Particles
}

\author{
Sujala Bhattarai ${ }^{1}$, Dae Hyun $\mathrm{Kim}^{1}{ }^{1}$, Jae-Heun $\mathrm{Oh}^{2}$ \\ ${ }^{1}$ Department of Biosystems Engineering, Kangwon National University, Chuncheon, Korea, \\ ${ }^{2}$ Forest Practice Research Center, Korea Forest Research Institute, Pocheon, Korea \\ Received: March 26 ${ }^{\text {th }}, 2012$; Revised: April 22 ${ }^{\text {th }}, 2012$; Accepted: April 27 ${ }^{\text {th }}, 2012$
}

\section{Abstract}

Purpose: The simulation model of a pneumatic conveying drying (PCD) for sawdust was developed and verified with the experiments. Method: The thermal behavior and mass transfer of a PCD were modeled and investigated by comparing the experimental results given by a reference (Kamei et al. 1952) to validate the model. Momentum, energy and mass balance, one dimensional first order ordinary differential equations, were coded and solved into Matlab V. 7.1.0 (2009). Results: The simulation results showed that the moisture content reduced from 194\% to 40\% (dry basis), air temperature decreased from $512^{\circ} \mathrm{C}$ to $128^{\circ} \mathrm{C}$ with the particle residence time of 0.7 seconds. The statistical indicators, root mean square error and R-squared, were calculated to be 0.079 , and 0.998 , respectively, between the measured and predicted values of moisture content. The relative error between the measured and predicted values of the final pressured drop, air temperature, and air velocity were only $8.96 \%, 0.39 \%$ and $1.05 \%$ respectively. Conclusions: The predicted moisture content, final temperature, and pressure drop values were in good agreement with the experimental results. The developed model can be used for design and estimation of PCD system for drying of wood dust particles.

Keywords: Pneumatic conveying drying, Simulation, Model validation, Sawdust

\section{Introduction}

Sawdust is the preferred materials for pellet production. The initial moisture content of the wet sawdust used for the pellet production is around $40 \%$ (dry basis, $\mathrm{db}$ ). However, Korean pellet manufactures generally dry the raw sawdust to around $15 \%$ of moisture content. The various types of dryers such as gas fuel dryer, steam dryers and rotary dryers, have been used for the sawdust drying.

Pneumatic conveying drying (PCD) also known as the flash drying is one of the most widely used technology for sawdust drying. This is the integration of the heat and mass transfer and pneumatic conveying technology. PCD is simple in construction, has low capital cost and short

\footnotetext{
*Comesponding author: Dae Hyun Kim

Tel: +82-33-250-6496; Fax: +82-33-255-6406

E-mail: daekim@kangwon.ac.kr
}

residence time. It allows higher inlet temperature than many other dryers without unduly heating of the products (Baeyens et al., 1995).

While drying sawdust, large amount of volatile organic compounds (VOC) is emitted to the atmosphere, which contribute to the formation of harmful photo-oxidants and also decrease the energy value of the sawdust (Lehtikangas, 2001). VOC emissions rate varies with the type of dryers; it depends upon the residence time, temperature and drying medium. VOC emissions increase with the particle residence time (Stahl et al., 2004), therefore with the advantage of having very short residence time, PCD could minimize the VOC emission, improve the energy content of the sawdust and also decrease environmental pollution.

Several studies have been performed both mathematically and experimentally to study the PCD performance of the various biomaterials and minerals such as potato (Pelegrian 
et al., 2001), grains (Matsumoto and Pei, 1984), rice (Tanaka et al., 2008 (a)), sludge (Jamaleddine and Ray, 2011) and alumina (Narimatsu et al., 2011). Kamei et al. (1952) reported the experimental study of pneumatic drying of sawdust particles in the vertical steel pipe with $0.1 \mathrm{~m}$ diameter and $14.5 \mathrm{~m}$ length; however, numerical approach of PCD for sawdust was limited. In the present study, the simulation of the vertical PCD was carried out to dry the sawdust particles and validated with the previous experimental results given by Kamei et al. (1952).

\section{Materials and Methods}

\section{System description and model development}

The descriptions of the systems used by the Kamie et al. (1952) for the experiments are explained as follows. The vertical PCD consists of a combustion chamber, hopper, table feeder, drying column, and cyclone. The drying column is a steel pipe with an inner diameter of $0.1 \mathrm{~m}$ and length of $14.5 \mathrm{~m}$. A hopper is used to carry sawdust before feeding to the screw feeder, which feeds the sawdust into the drying column. Hot air from the combustion chamber is blown to convey and dry the sawdust. A cyclone is used to separate the sawdust particles and the hot air. A schematic diagram of a PCD of sawdust is shown in Fig. 1.

(Kamei et al., 1952)

The energy balance equations given by Tanaka et al. (2008 (b)) (derived for PCD of rice) and Pelegrina and Crapiste (2000) (derived for PCD of potato) were modified and used for the simulation study for drying of sawdust. In contrast to the previous developed models, the relationships determining the physical properties (density, specific heat capacity, specific gravity) of soft wood materials were used in the present models. Similarly, the water activity equation of softwood particles was employed to determine the relationship between the water activity and the particle temperature.

The model was developed from momentum, energy and mass balances by employing constitutive heat and mass transfer relationships between the sawdust particles and drying air for simulating sawdust drying rate, temperature change and moisture contents. Assuming the up-stream air flow and spherical shape of the particles, equations were solved in the Matlab v.7.0.4. (Mathworks, Inc., 2011). Inlet air velocity, moisture content, humidity, air temperature and particle temperature were used to specify the initial values for the equations. All the initial parameters were taken same as that given by Kamei et al. (1952) as shown in Table 1.

The momentum balance equation for the sawdust particle is expressed as:

$$
v_{p} \frac{d v_{p}}{d z}=\frac{3}{4 d_{p}} C_{D} \frac{\rho_{a}}{\rho_{p}}\left(v_{a}-v_{p}\right)^{2}-\left(1-\frac{\rho_{a}}{\rho_{p}}\right) g-f_{p}\left(\frac{v^{2} p}{2}\right)\left(\frac{1}{D}\right)(1)
$$

The first, second and third terms in the right hand side of equation (1) represent the motion due to the drag force, gravitational force, and frictional force respectively.

The momentum balance equation for the air is given as:

$$
\begin{aligned}
v_{a} \frac{d v_{a}}{d z}= & -\frac{1}{\rho_{a}} \frac{d P}{d z}-g-4 f\left(\frac{v_{a}^{2}}{2}\right)\left(\frac{1}{D}\right) \\
& -\frac{3}{4 d_{p}}\left(\frac{1-\epsilon}{\epsilon}\right) C_{D}\left(v_{a}-v_{p}\right)^{2}-\frac{6 \dot{w}}{\rho_{a} d_{p}}\left(\frac{1-\epsilon}{\epsilon}\right)\left(v_{a}-v_{p}\right)
\end{aligned}
$$

Each term from the right hand side in the equation represents the resistance in the air velocity due to the pressure drop, frictional force, drag force and mass transfer respectively. The pressure drop along the length of the dryer is expressed as:

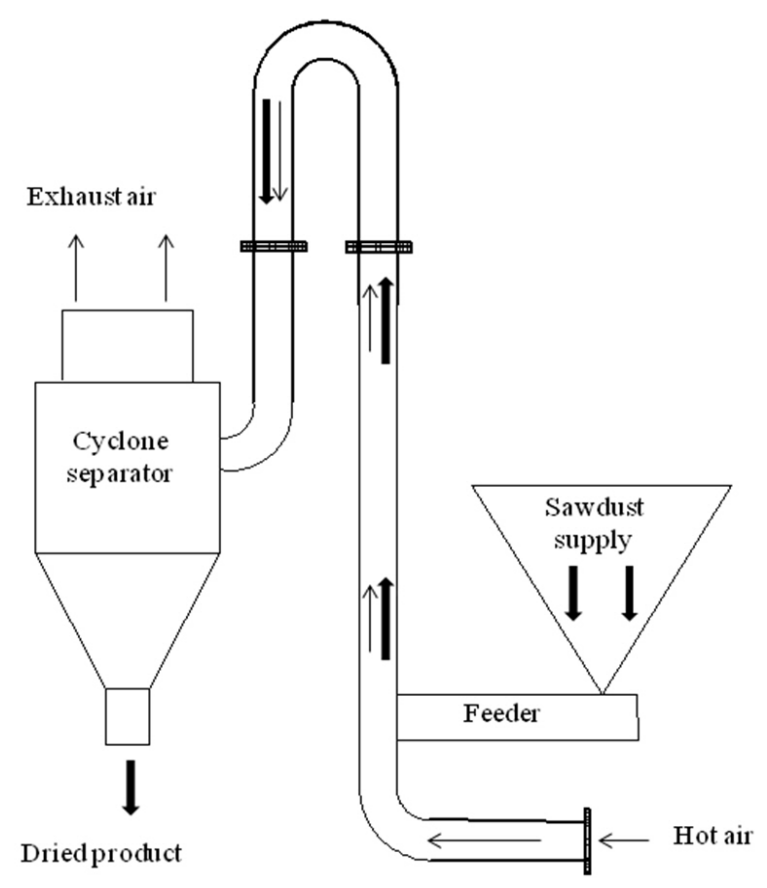

Figure 1. Schematic of the vertical pneumatic dryer. 
$\frac{d P}{d z}=\left(\epsilon \rho_{a}+(1-\epsilon) \rho_{p}\right) g+(1-\epsilon) \rho_{p} v_{p} \frac{d v_{p}}{d Z}+F_{p}+F_{a}$

The frictional forces, $F_{a}$ and $F_{p}$, can be calculated using the following expressions:

$$
\begin{aligned}
& F_{a}=f \rho_{a} \epsilon \frac{v_{a}^{2}}{2 D} \\
& F_{p}=f_{p}(1-\epsilon) \rho_{p} \frac{v_{p}^{2}}{2 D}
\end{aligned}
$$

Where $f$ and $f_{p}$ represent the frictional factors and $D$ represents the column diameter.

Where Volume fraction is written as:

$$
\frac{1-\epsilon}{\epsilon}=\frac{\rho_{a} v_{a} G_{d p}(1+M)}{\rho_{p} v_{p} G_{d a}(1+H)}
$$

The energy balance equation for the particle can be written as:

$$
v_{p} \frac{d T_{p}}{d z}=\frac{6}{d_{p} \rho_{p} C_{p}}\left(h\left(T_{a}-T_{p}\right)-\dot{w} q\left(T_{p}, M\right)\right)
$$

Where, $h$ is the convective heat transfer coefficient between the hot air and the particles and $\dot{w}$ is the mass transfer coefficient.

The energy balance equation for the air is given as:

$$
\begin{aligned}
& v_{a} \frac{d T_{a}}{d z}=-\frac{6 h}{d_{p} \rho_{a} C_{a}}\left(\frac{1-\epsilon}{\epsilon}\right)\left(T_{a}-T_{p}\right) \\
& -\frac{6 \dot{w} C_{v}}{d_{p} \rho_{a} C_{a}}\left(\frac{1-\epsilon}{\epsilon}\right)\left(T_{a}-T_{p}\right)-\frac{Q}{G_{d a} C_{a}(1+H)} \\
& Q=\pi \epsilon D U\left(T_{a}-T_{a m}\right)
\end{aligned}
$$

The first, second and third terms in the right hand side in equation (8) represent the energy loss by air due to convection, mass transfer and the surroundings respectively.

The mass balance equation for the particle phase is written as:

$$
\frac{d M}{d z}=-\frac{6 \dot{w}(1+M)}{d_{p} \rho_{p} v_{p}}
$$

The mass balance equation for the air humidity is expressed as:

$$
\frac{d H}{d z}=-\left(\frac{G_{d p}}{G_{d a}}\right)\left(\frac{d M}{d z}\right)
$$

Where, $G_{d p}$ and $G_{d a}$ are the mass flow rate of dry matter of sawdust particle and dry air, respectively.

\section{Complementary equations}

\section{Solid properties}

Pinewood sawdust was used for the simulation study. The solid properties were calculated from the proposed equations for the pine wood (Younsi et al., 2006).

Density

$\rho=1000 G_{m}(1+M)$

Where, $G_{m}$ is specific gravity of wood.

Specific heat

$$
C_{p}=\left[\frac{C_{p o}+0.01 C_{p w} M}{1+0.01 M}\right]+A_{c}
$$

Where $M$ is the moisture content (\%), $C_{p w}$ is the heat capacity of water, $A_{c}$ is a parameter, which is a function of moisture content and temperature and $C_{p o}$ is the heat capacity of the dry wood. $C_{p o}$ and $A_{c}$ are as below:

$$
\begin{aligned}
& A_{c}=M\left(-0.06191+2.36 \times 10^{4} T_{p}-1.33 \times 10^{-4} M\right) \\
& C_{p o}=0.1031+0.0038 T_{p}
\end{aligned}
$$

Drag coefficient

$$
\begin{array}{ll}
C_{D}=\frac{24}{R e_{p}}\left(1+0.15 R e_{p}^{0.687}\right) & R e_{p} \leq 1000 \\
C_{D}=0.44 & R e_{p}>1000
\end{array}
$$

Where $C_{D}$ is the drag coefficient and $R e_{p}$ is the particle Reynolds number. $R e_{p}$ is given below:

$$
R e_{p}=\frac{\left|v_{p}-v_{a}\right| d_{p} \rho_{a}}{\eta_{a}}
$$


To calculate the convective heat transfer coefficient between the air and particle, Ranz-Marshall equation was used (Pelegrina and Crapiste, 2000) which is expressed as:

$$
h=\frac{\lambda_{a}\left(2+0.6 \operatorname{Pr}^{\frac{1}{3}} R e^{\frac{1}{2}}\right)}{d p}
$$

Mass transfer coefficient between air and particle (Tanaka et al, 1950 (a))

$$
\dot{w}=k_{m}\left[0.622 \frac{a_{w} P_{s a t}\left(T_{p}\right)}{P-a_{w} P_{s a t}\left(T_{p}\right)}-H\right]
$$

The relationship between heat and mass transfer can be related as follows:

$$
\begin{aligned}
k_{m}= & \frac{h}{C_{a}(1+H)\left(\frac{\alpha}{D_{v}}\right)^{\frac{2}{3}}} \\
D_{v}= & 2.35524 \times 10^{-10} T_{a}^{2}+9.39585 \times 10^{-9} T_{a} \\
& +1.651947 \times 10^{-6}
\end{aligned}
$$

Here, $D_{v}$ is the diffusion of water vapor in air, and $h$ is the convective heat transfer coefficient.

Latent heat of vaporization of water (Tanaka et al., 2008 (b))

$$
\begin{aligned}
& q\left(T_{p}, M\right)=\left(V_{v}-V_{1}\right) T_{p}\left(\frac{d p_{p}\left(T_{p}, M\right)}{d T_{p}}\right)_{v} \\
& V_{v}-V_{1}=\frac{461.5 T_{p}}{P_{p}\left(T_{p}, M\right)}-0.001 \\
& p_{p}\left(T_{p}-T\right)=a_{w}\left(T_{p}, M\right) p_{s a t}\left(T_{p}\right) \\
& \left(\frac{d p_{p}\left(T_{p}, M\right)}{d T_{p}}\right)= \\
& \left(\frac{d a_{w}\left(T_{p}, M\right)}{d T_{p}}\right) p_{s a t}\left(T_{p}\right) \\
& +a_{w}\left(T_{p}, M\right)\left(\frac{d p_{s a t}\left(T_{p}\right)}{d T}\right)_{v}
\end{aligned}
$$

Where $a_{w}$ is the water activity given by Pakowski et al. (2007) for the softwood. The latent heat of vaporization of water in rice particles was used for sawdust.

Frictional factors

Frictional factor between the air and the wall (Tanaka et al., 2008)

$$
\begin{array}{ll}
f=\frac{16}{R e} & R e \leq 2300 \\
\frac{1}{\sqrt{f}}=3.2 \log \operatorname{Re} \sqrt{f}+1.2 & R e>2300
\end{array}
$$

Where, $R e$ is the Reynolds number expressed as:

$$
R e=\frac{\rho_{a} v_{a} D}{\eta_{a}}
$$

Frictional factor for the wall-particle interaction is given by (Han et al., 2000)

$$
f_{p}=1.0503 F r_{p}^{-1.831}
$$

Where the particle Froude number $F r_{p}$ is written as (Han et al., 2000)

$$
F r_{p}=v_{p} /\left(g d_{p}\right)^{0.5}
$$

The residence time of the particles at the gas phase, $t_{p}$, was calculated by:

$$
\frac{d t_{p}}{d x}=\frac{1}{v_{p}}
$$

\section{Statistical indicators}

Root mean square error (RMSE) was used as one of the statistical indicators. The results are better with lower values of $\mathrm{RMSE}_{1}$ but few large errors in the sum can produce a significant increase of $\mathrm{RMSE}_{1}$. Therefore, $\mathrm{RMSE}_{2}$ was developed eliminating the drawbacks of $\mathrm{RMSE}_{1}$. These follow the dimension of the predicted quantities. $\mathrm{RMSE}_{1}$ and $\mathrm{RMSE}_{2}$ can be expressed as (Stone, 1993 and Kim et al., 2007)

$$
\begin{aligned}
& \operatorname{RMSE}_{1}=\left(\frac{1}{m} \sum_{j=1}^{m} d_{j}^{2}\right)^{\frac{1}{2}} \\
& \operatorname{RMSE}_{2}=\left(\frac{1}{m} \sum_{j=1}^{m}\left(\frac{d_{j}}{Y_{j}}\right)^{2}\right)^{\frac{1}{2}}
\end{aligned}
$$

$d_{j}$ is the deviation between the $\mathrm{j}^{\text {th }}$ measured and the predicted values, $m$ is the number of data points and $Y_{j}$ is the $\mathrm{j}^{\text {th }}$ measured value. $\mathrm{R}$-squared is a statistical measure of how well a predicted line approximates measured data. R-squared value equal to 1 implies that the model 
provides perfect prediction, and 0 implies that there is no relationship between the measured and predicted value. This can be expressed as (Stone, 1993 and Kim et al., 2007)

$$
\text { R-squared }=\left[\frac{m\left(\sum_{j=1}^{m} X_{j} Y_{j}\right)-\left(\sum_{j=1}^{m} X_{j}\right)\left(\sum_{j=1}^{m} Y_{j}\right)}{\sqrt{\left[m \sum_{j=1}^{m} X_{j}^{2}-\left(\sum_{j=1}^{m} X_{j}\right)^{2}\right]\left[m \sum_{j=1}^{m} Y_{j}^{2}-\left(\sum_{j=1}^{m} Y_{j}\right)^{2}\right]}}\right]^{2}
$$

Where $X_{j}$ is the $\mathrm{j}^{\text {th }}$ predicted value.

\section{Results and Discussion}

\section{Simulation results}

The input data used for the simulation study is displayed in Table 1. The simulation was done with the particles size of $0.003 \mathrm{~m}$ diameter. Fig. 2 to 6 show the simulated particle velocity, gas velocity, moisture content of particle, air humidity, particle temperature, air temperature, air pressure, and slip velocity along the riser length.

The predicted sawdust particles temperature and hot air temperature along the length of the drying column is shown in Fig. 2 (a) and (b) respectively. In general, air temperature decreased and particle temperature increased along the dryer length. Particles receive heat from the air due to the convection heat transfer between the particles and air which increased the particle temperature (Fig. 2 (a)). Air temperature decreased from $512.0^{\circ} \mathrm{C}$ to $128.5^{\circ} \mathrm{C}$ by the end point of the vertical PCD because sensible heat of the air is utilized for drying (Fig. 2 (b)).

The particle velocity, air velocity and particle slip velocity are shown in Fig. 3 (a), (b) and (c), respectively.

\section{Table 1. Parameters used for simulation}

\begin{tabular}{|cc|} 
Parameters & Initial values \\
\hline Particle temperature $\left({ }^{\circ} \mathrm{C}\right)$ & 25 \\
\hline Air temperature $\left({ }^{\circ} \mathrm{C}\right)$ & 515 \\
\hline Air velocity $\left(\mathrm{m} \mathrm{s}^{-1}\right)$ & 42 \\
Particle velocity $\left(\mathrm{m} \mathrm{s}^{-1}\right)$ & 0 \\
\hline Air humidity $\left(\mathrm{kg} \mathrm{kg}^{-1}\right)$ & 0.019 \\
Moisture content $(\%, \mathrm{db})$ & 194 \\
Feed rate $\left(\mathrm{kg} \mathrm{h}^{-1}\right)$ & 137 \\
\hline Air flow rate $(\mathrm{kg} \mathrm{dry} \mathrm{gas} \mathrm{h})$ & 633 \\
\hline
\end{tabular}

Slip velocity also known as relative velocity is the difference between the air velocity and the particle velocity. Air velocity decreased along the length of dryer, from 42.00 to $35.47 \mathrm{~m} \mathrm{~s}^{-1}$, due to increased air density caused by reduction in air temperature. Particle velocity increased due to the higher air velocity. At the end of the drying, particle velocity and air velocity was found almost equal. The slip velocity was highest at the feed point, near about $40 \mathrm{~m} \mathrm{~s}^{-1}$, and decrease towards the column length to about $10 \mathrm{~m} \mathrm{~s}^{-1}$ by the end point. This is likely due to the acceleration of solid particles prior to attaining the certain velocity, which result in higher solid holdup and in turn higher slip velocity at the bottom of the dryer.

The predicted particle moisture content and air humidity are shown in Fig. 4 (a) and (b) respectively. The moisture content decreased from 194\% to about 39\%
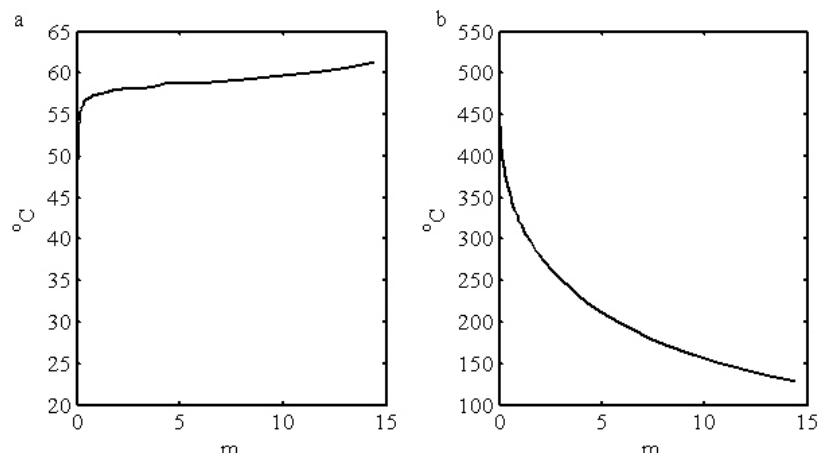

Figure 2. The predicted temperature of (a) sawdust particles and (b) hot air along the dryer length.
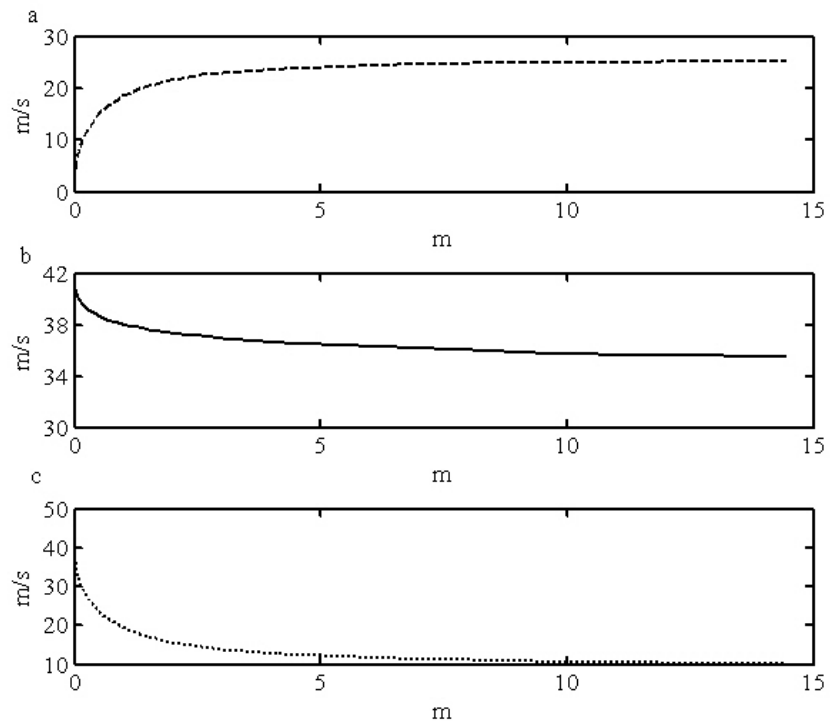

Figure 3. The predicted (a) particle velocity (b) air velocity and (c) slip velocity along the dryer length. 
after drying in the vertical PCD of 14.7 m length. Meanwhile, humidity shows the increasing trend. This increased rapidly near the inlet due to high slip velocity between the air and the solid particles.

The change in pressure throughout the length of the vertical PCD is shown in Fig. 5. It can be seen from the Fig. 5 that there was a total pressure drop of $701 \mathrm{~Pa}$. Pressure decreased along the length of the vertical tube due to the weight of the solid particles (Yerushalmi and Cankurt, 1979) and decrease of the air velocity (Rautiainen et al., 1999).

The particle velocity with respect to its residence time is shown in Fig. 6. It can be seen that the particle stayed about 0.7 seconds inside the PCD system. Thus, from PCD technology sawdust could be dried in very short time to the target moisture content.

\section{Model validation}

The experimental data given by Kamei et al. (1952)
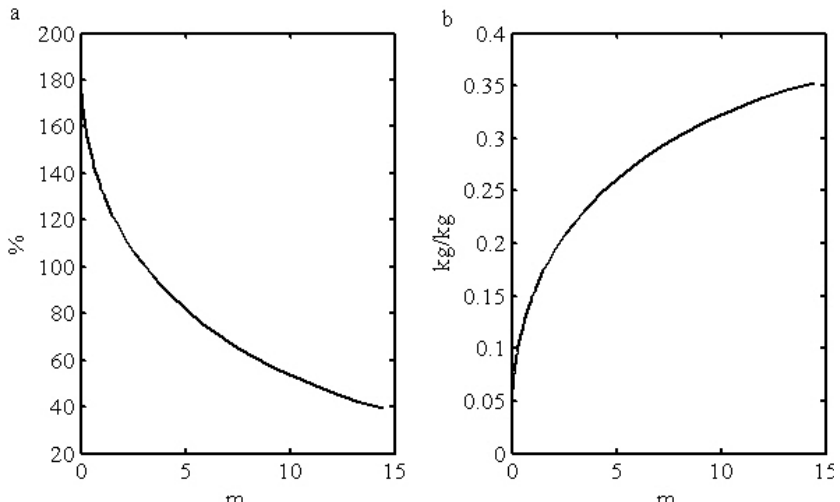

Figure 4. The predicted (a) moisture content of sawdust particles and (b) humidity of hot air along the dryer length.

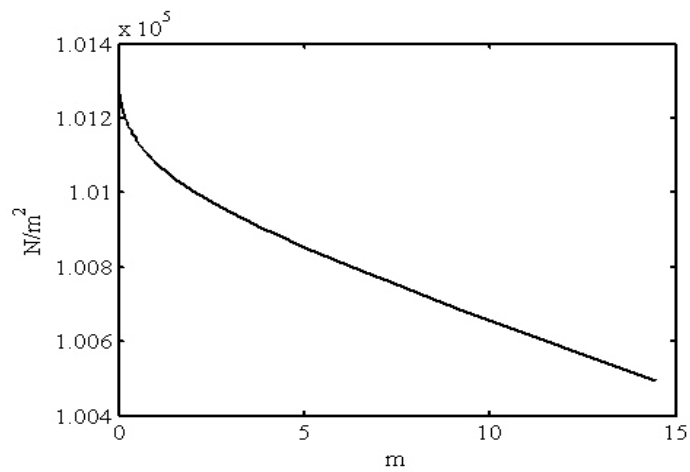

Figure 5. The predicted pressure drop along the length of the dryer. was used for the validation of developed model for the sawdust drying. Measured and predicted values of total pressure drop, final air temperature and gas velocity at the end point of the vertical column are shown in Table 2. We can see that the all the predicted data are in good agreement with the experimental values. Relative error between the measured and predicted values of total pressure drop, final air temperature, and final gas velocity were calculated as $8.96 \%, 0.39 \%$ and $1.05 \%$, respectively.

The moisture content of the sawdust particles derived from simulation study and the experiments of Kamei et al. (1952) is shown in Fig. 7. The predicted moisture content is in good agreement with the experimental results. There are no any apparent bios between the measured and predicted moisture content (Fig. 8). The statistical

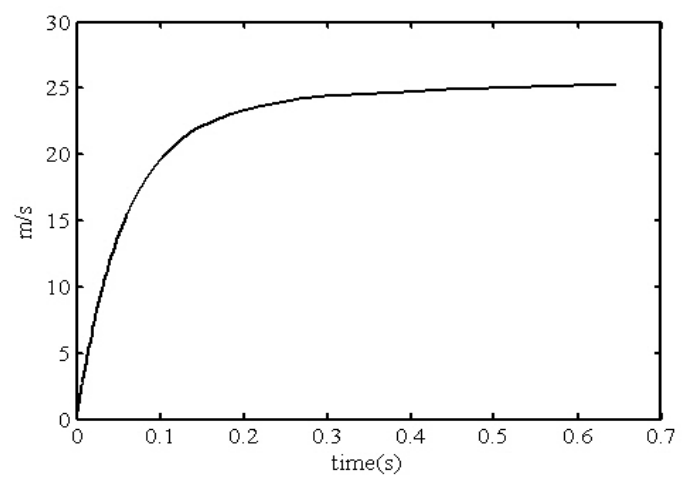

Figure 6. The predicted particle velocity with respect to the particle residence time.

Table 2. Measured and predicted values of different parameters of PCD

\begin{tabular}{|cccc|} 
& $\begin{array}{c}\text { Total pressure } \\
\text { drop }(\mathrm{Pa})\end{array}$ & $\begin{array}{c}\text { Final air temperature } \\
\left({ }^{\circ} \mathrm{C}\right)\end{array}$ & $\begin{array}{c}\text { Air velocity } \\
\left(\mathrm{m} \mathrm{s}^{-1}\right)\end{array}$ \\
\hline Predicted & 701 & 128.5 & 35.47 \\
Measured & 770 & 128.0 & 35.10 \\
\hline
\end{tabular}

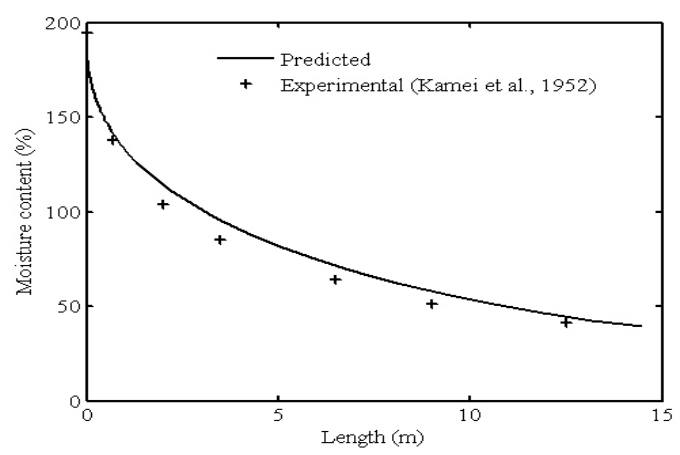

Figure 7. Measured and predicted moisture content of sawdust. 


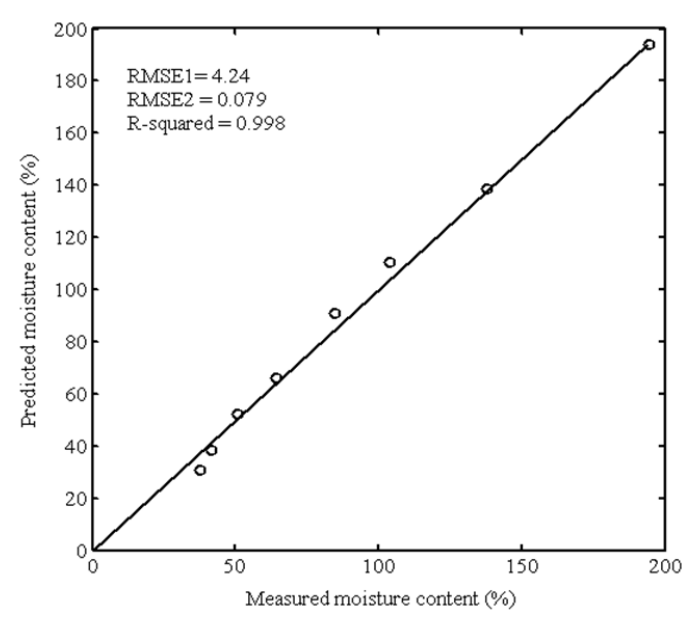

Figure 8. Measured and predicted moisture content of sawdust with statistical indicators.

indicators $\mathrm{RMSE}_{1}, \mathrm{RMSE}_{2}$, and R-squared were obtained as $4.240,0.079$, and 0.998 respectively.

\section{Conclusions}

Pneumatic conveying drying models were developed to simulate the collective behavior of wood dust particles in moist air flow. The model takes into account the momentum, heat and mass transfer between the air and the particle. The model was validated against pneumatic transport and pneumatic drying experimental results for sawdust. Statistical indicators, $\mathrm{RMSE}_{1}, \mathrm{RMSE}_{2}$, and Rsquared values, were also calculated to define relationship between the moisture content of measured and the predicted values. The relative error between the measured and predicted temperature, moisture content and pressure drop were also found in the acceptable range. The predicted values were in good agreement with the experimental results. The particle residence time was found near about 0.7 seconds. The simulated equation could be used for the preliminary design estimation for pneumatic drying of sawdust particles.

\section{Conflict of Interest}

No potential conflict of interest relevant to this article was reported.

\section{References}

Baeyens J., D. V. Gauwbergen and L. Vinckier. 1995. Pneumatic Drying: The use of large scale data in the design procedure. Powder Technology 83:139-148.

Han, T., A. Levy and Y. Peng. 2000. Model for dilute gas particle flow in constant area lance with heating and friction. Powder technology 112:283-288.

Jamaleddine, J. T. and M. B. Ray. 2011. Drying of Sludge in a Pneumatic Dryer Using Computational Fluid Dynamics. Drying Technology 29(3):308-322.

Kamei S. and R. Toei. 1952. Studies on pneumatic conveying drying. Kagaku Kogaku 16:297-300. (In Japanese, with English abstract).

Kim, D. H., B. M. Jenkins, T. R. Rumsey, M. W. Yore and N. J. Kim. 2007 Simulation and model validation of a horizontal shallow basin solar concentrator, Solar Energy 81: 463-475.

Lehtikangas, P. 2001. Quality properties of pelletised sawdust, logging residuals and bark. Biomass and Bioenergy 20:351-360.

Matsumoto, S. and D. C. T. Pei. 1984. A mathematical analysis of pneumatic drying of grains-I. Constant drying rate, Journal of heat and mass transfer, 27(6):743-849.

Narimatsu, C. P., M. C. Ferreira and J. T. Freire. 2007. Drying of Coarse Particles in a Vertical Pneumatic Conveyor. Drying Technology 25(2):291-302.

Pakowski, Z., B. Krupinska and R. Adamski. 2007. Prediction of sorption equilibrium both in air and superheated steam drying of energetic variety of Willo Salix Viminalis in a wide temperature range. Fuel 1749-1757.

Pelegrina, A. H. and G. H. Crapiste. 2000. Modelling the pneumatic drying of food particles. Journal of Food Engineering 48:301-310.

Stahl, M., K. Granstrom, J. Berghel and R. Renstrom. 2004. Industrial processes for biomass drying and their effects on the quality properties of wood pellets. Biomass and Bioenergy 27:621-628.

Stone, R. J. 1993. Improved statistical procedure for the evaluation of solar radiation estimation models, Solar Energy 51(4):289-291.

Tanaka, F., T. Uchino, D. Hamanaka and G. G. Atungulu. 2008. Mathematical modeling of pneumatic drying of rice powder. Journal of food Engineering 88:492-498.

Tanaka, F., Y. Maeda, T. Uchino, D. Hamanaka and G. G. Atungulu. 2008 (a). Monte Carlo simulation of the collective behavior of food particles in pneumatic drying 
operation. Food science and technology 41: 1567-1574.

Tanaka, F., Y. Maeda, T. Uchino, D. Hamanaka and G. G. Atungulu. 2008 (b). Monte Carlo simulation of the collective behavior of food particles in pneumatic drying operation. Food science and technology 41: 1567-1574.

Yerushalmi, J. and N. T. Cankurt. 1979. Further Studies on the regimes of Fluidization. Powder Technology 24: 187- 205.

Younsi, R., D. Kocaefe, S. Poncsak and Y. Kocaefe. 2006. A diffusion model for transient high temperature treatment of wood. Journal of Building Physics 30:113.

\section{Nomenclature}

$\begin{array}{ll}A_{c} & \text { dimensionless parameter } \\ a_{w} & \text { water activity (dimensionless) } \\ C & \text { specific heat capacity }\left(\mathrm{J} \mathrm{kg}^{-1} \mathrm{~K}^{-1}\right) \\ C_{D} & \text { drag coefficient (decimal) } \\ C_{p o} & \text { specific heat of dry wood }\left(\mathrm{kJ} \mathrm{kg}^{-1} \mathrm{~K}^{-1}\right) \\ C_{p w} & \text { specific heat of water }\left(\mathrm{kJ} \mathrm{kg}^{-1} \mathrm{~K}^{-1}\right) \\ d & \text { deviation (m) } \\ d p & \text { particle diameter }(\mathrm{m}) \\ D & \text { pipe diameter }(\mathrm{m}) \\ D_{v} & \text { diffusion of water vapor in air, }\left(\mathrm{m}^{2} \mathrm{~s}^{-1}\right) \\ f & \text { frictional coefficient }(\mathrm{decimal}) \\ F & \text { frictional force }\left(\mathrm{kg} \mathrm{s} \mathrm{m}^{-2}\right) \\ F r_{p} & \text { Froude number } \\ G & \text { mass flow rate of air }\left(\mathrm{kg} \mathrm{s}^{-1}\right) \\ g & \text { gravitational constant }\left(\mathrm{m} \mathrm{s}^{-2}\right) \\ G_{m} & \text { specific gravity (dimensionless) } \\ H & \text { absolute humidity }\left(\mathrm{kg} \mathrm{kg}^{-1}\right) \\ h & \text { convective heat transfer coefficient }\left(\mathrm{w} \mathrm{m}^{-2} \mathrm{~K}^{-1}\right)\end{array}$

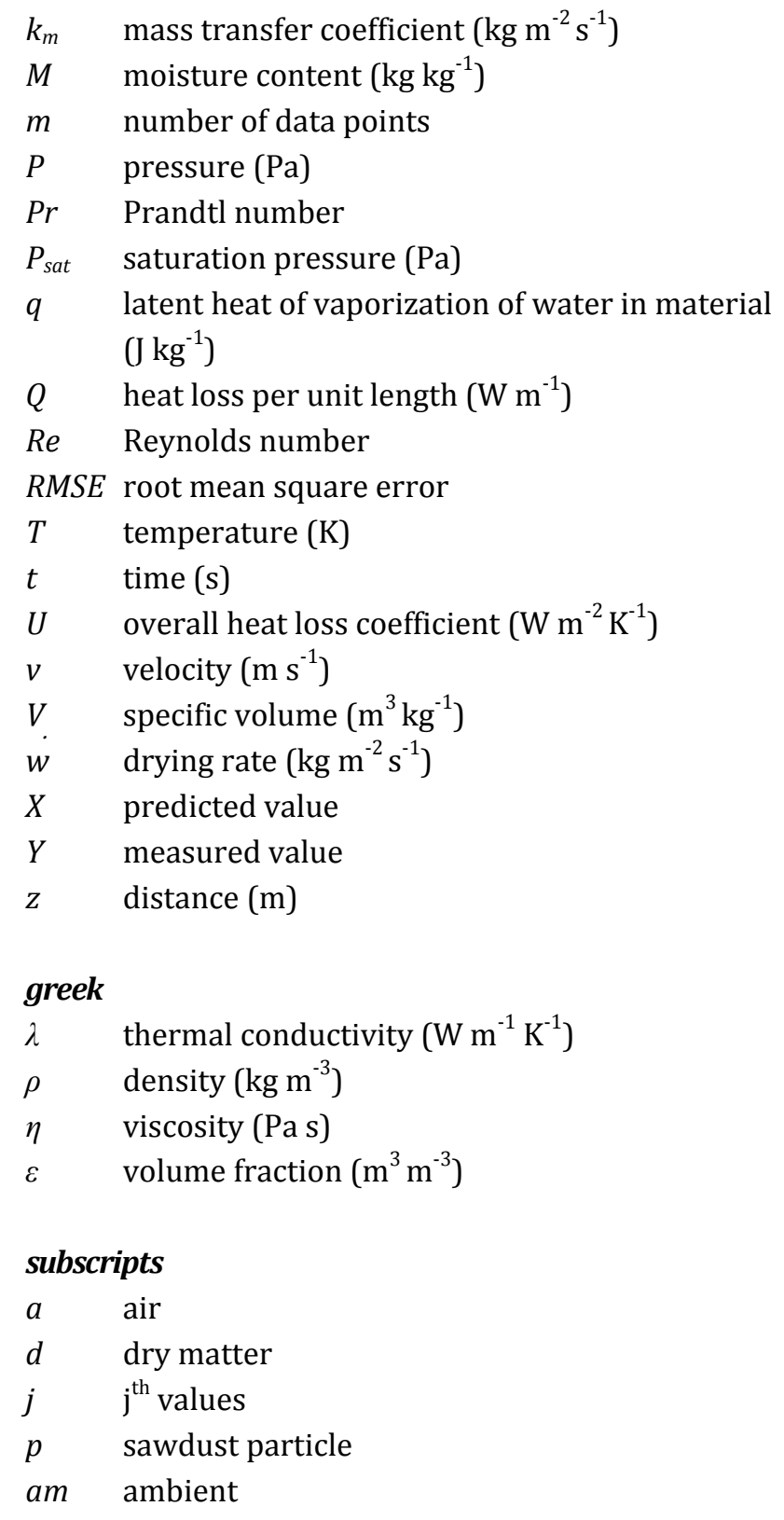

